\title{
Réponses gliales et vasculaires à la mort neuronale d'origine excitotoxique
}

L'évolution vers la cicatrice gliale d'une lésion cérébrale provoquée par l'injection d'une substance excitotoxique se fait en trois phases. Pendant les 24 ou 48 premières heures, la mort neuronale est associée à des modifications de tous les éléments de la zone lésée, aboutissant à une rupture de la barrière hémato-encéphalique. A la faveur de cette rupture, des cellules et des protéines normalement exclues du système nerveux central pénètrent la lésion qui est le siège d'une intense prolifération cellulaire, notamment d'origine monocytaire et microgliale. Parallèlement, les terminaisons axonales qui contactaient les neurones disparus bourgeonnent et constituent des cônes de régénération. Après le premier mois, une cicatrice gliale astrocytaire s'établit aux dépens des autres espèces cellulaires. C'est au cours de la seconde phase qu'existent probablement les meilleures chances de récupération après greffe de cellules fotales.

Isabelle Dusart

Serge Marty Marc Peschanski

\section{ADRESSES}

I. Dusart : étudiante post-doctorale, université de Zurich, Confédération helvétique.

S. Marty : boursier du ministère de la Recherche et de la Technologie.

M. Peschanski : direcleur de recherche à l'Inserm, Inserm CJF 91-02, service de médecine nucléaire, centre hospitalier universitaire Henri-Mondor, 94010 Créteil,

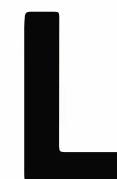

e système nerveux central des mammiferes adultes fonctionne dans le cadre d'interactions cellulaires entre neurones, cellules gliales et cellules endothéliales. Lors de lésions excitotoxiques provoquant la mort d'une population de neurones, cet équilibre est rompu. Il se produit alors, au cours des mois qui suivent, des modifications de tous les éléments cellulaires du tissu nerveux concerné. Nous avions notamment remarqué une modification de la morphologie des terminaisons axonales privées de leurs cibles suite à la destruction de neurones thalamiques par une excitotoxine [1] (l'acide kaïnique). Deux à trois semaines après la lésion, et pour une période de quelques mois, les terminaisons axonales qui contactaient préalablement les neurones détruits bourgeonnent, présentant une morphologie comparable à celle des cônes de croissance. Nous avons démontré la potentialité régénérative de ces bourgeonnements en montrant qu'ils peuvent former de nouveaux contacts synaptiques si on leur procure des cibles nouvelles sous la forme de neurones fotaux transplantés [2, 3]. 
Ces premiers résultats nous ont conduits à entreprendre une étude systématique de l'environnement cellulaire dans lequel apparaissent ces différences morphologiques des terminaisons axonales, à la recherche tant d'éléments qui pourraient les favoriser que de ceux qui, à terme, pourraient participer à leur élimination. Nous avons ainsi suivi l'évolution de tous les éléments gliaux et vasculaires présents dans le tissu lésionnel jusqu'à la formation de la cicatrice astrocytaire. Cette étude nous a permis de définir trois phases entre le moment de la mort neuronale et celui de la cicatrisation du tissu: une courte (24-48 heures) période de réaction des éléments cellulaires qui étaient présents avant que la lésion neuronale soit réalisée, puis une phase de plusieurs semaines caractérisée par le recrutement et la prolifération de nombreux types cellulaires, enfin l'établissement progressif de la cicatrice astrocytaire.

\section{Phase 1 : réaction des éléments en place avant la lésion}

Pendant 24 à 48 heures après l'injection d'acide kaïnique, la mort neuronale n'induit ni prolifération ni recrutement cellulaire. En revanche, les éléments gliaux et vasculaires présents dans la zone de lésion sont tous profondément modifiés.

Les cellules microgliales, considérées comme les macrophages résidents du cerveau*, présentent une série de modifications que l'on regroupe sous le terme d'" activation". Leur corps

* Voir l'article de M. Tardieu et al., p. 775. cellulaire s'élargit, leurs prolongements s'épaississent et se raccourcissent, les sites de liaison membranaire de l'isolectine B4 tirée de Griffonia simplicifolia - utilisée comme marqueur de ces cellules [4] - sont nettement plus nombreux.

Les astrocytes sont des cellules étoilées qui contiennent des gliofilaments dont un des composants est la GFAP (glial fibrillary acidic protein)**. On peut donc marquer les astrocytes en utilisant un anticorps contre la GFAP. Après l'injection d'acide kaïnique, on observe un gonflement de ces cellules et la rétraction de leurs prolongements, associés à un accroissement du marquage immunocytochimique (figure 1). Dans notre cas, cet accroissement du marquage

* Voir l'article de M. Tardy, p. 799.

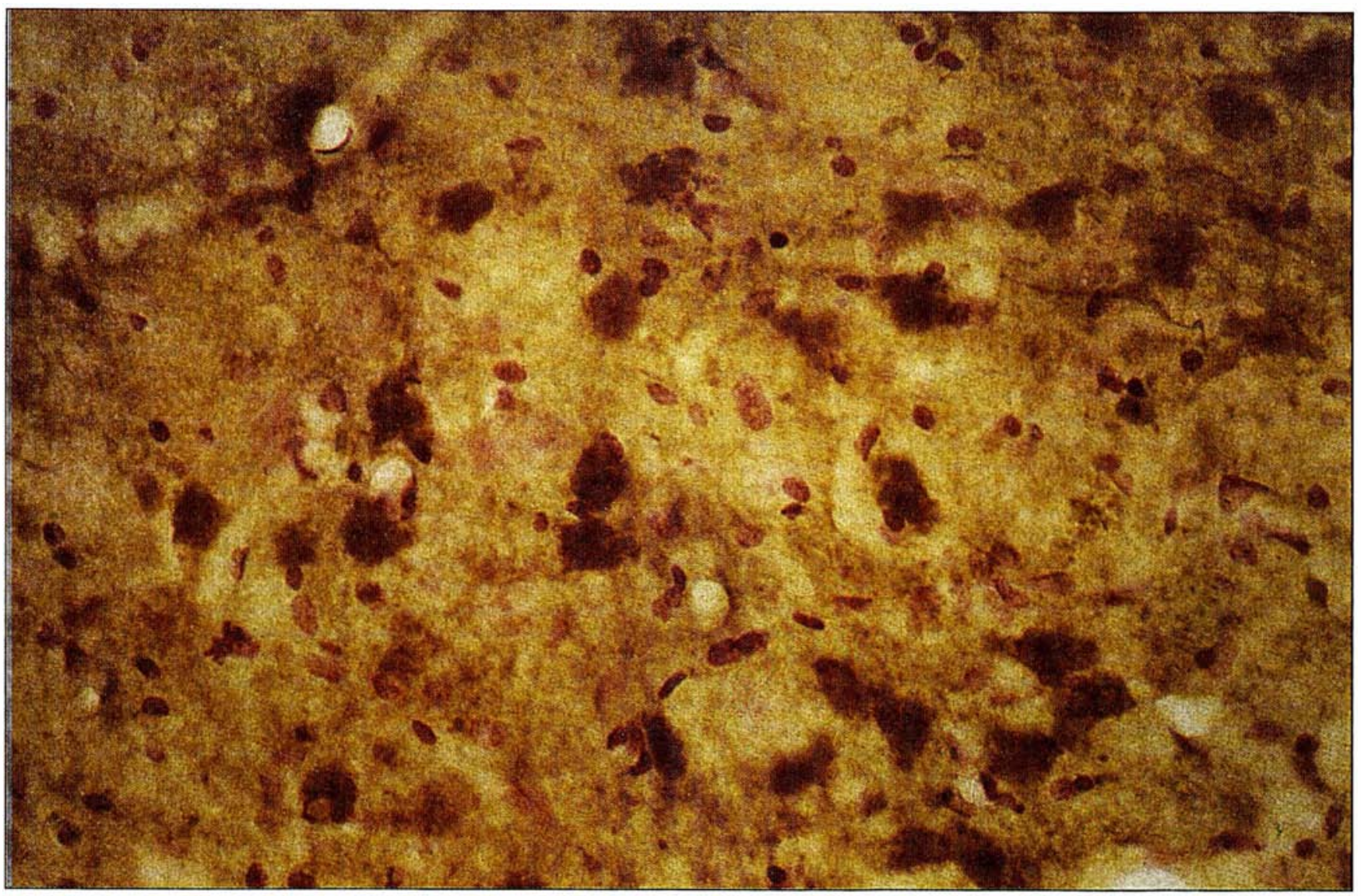

Figure 1. Astrocytes dans la zone déplétée en neurones 24 heures après l'injection d'acide kaïnique lles noyaux sont colorés au violet de crésyl, le marquage brun révèle la fixation de l'anticorps dirigé contre la GFAPI.

$\mathrm{m} / \mathrm{s} n^{\circ} 8$, vol. 7 , octobre 91 

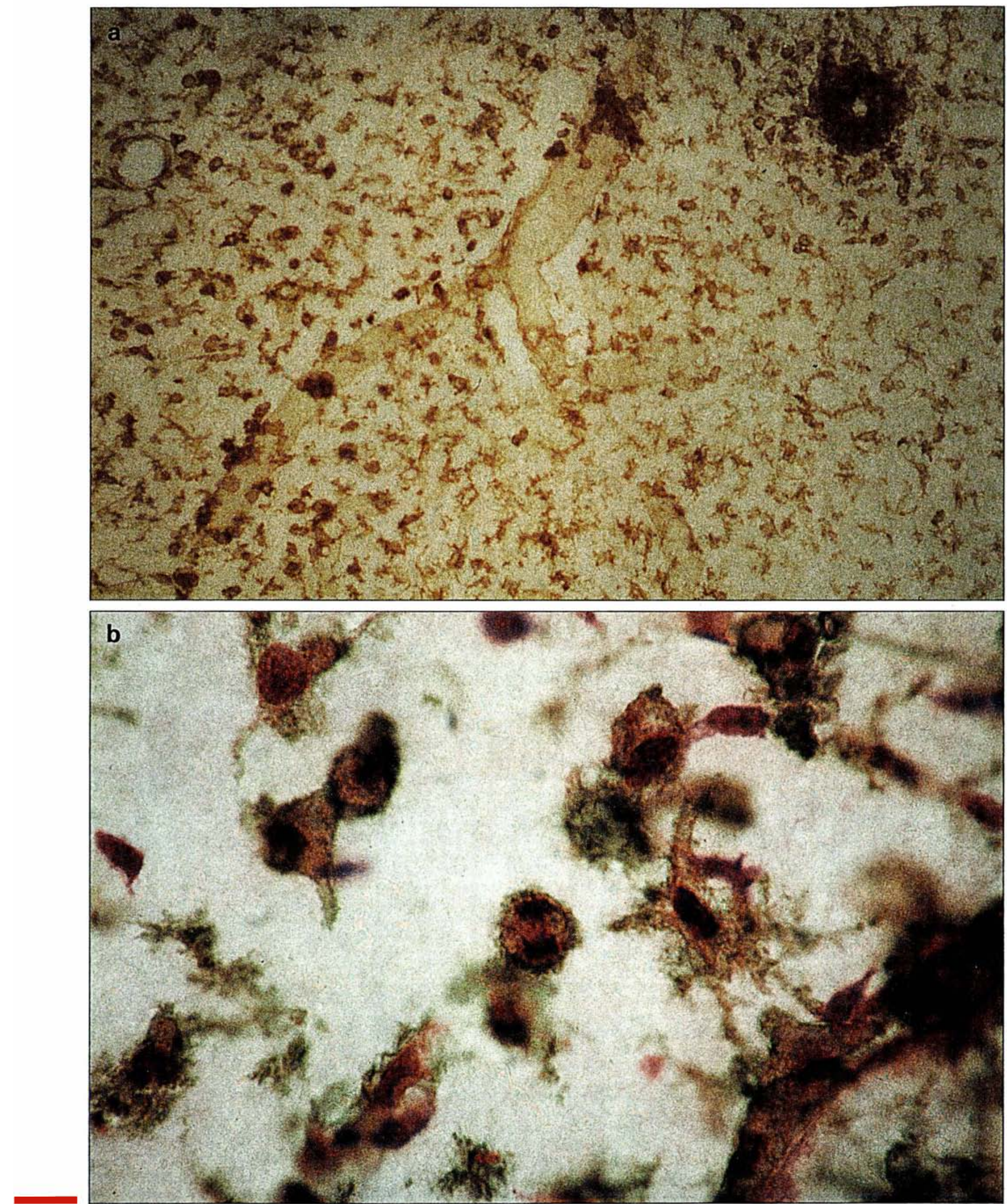
4 Figure 2. Réaction microgliale/macrophagique 4 jours après la lésion : a. amas de cellules globulaires marquées par la lectine autour de vaisseaux; b. cellule en mitose (les chromosomes sont colorés au violet de crésyl) marquée par la lectine (le marquage brun révèle la fixation de cette lectine).

immunocytochimique n'est pas le résultat d'une différenciation de ces cellules, mais celui de la désorganisation de leur cytosquelette.

Les astrocytes délimitent la frontière du système nerveux central en formant une glia limitans, notamment au niveau des vaisseaux sanguins. En effet, les prolongements astrocytaires font normalement une couche continue autour des vaisseaux sanguins cérébraux, en regard desquels ils forment une lame basale. Après une lésion excitotoxique, on observe en microscopie électronique une disparition des pieds astrocytaires formant la glia limitans. La frontière du système nerveux central est rompue. Enfin, cette première phase de réponse est marquée par l'ouverture de la barrière hémato-encéphalique ces circulantes qui peuvent - ou non - pénétrer dans le système nerveux central. Cette barrière, réalisée par les cellules endothéliales, pourrait être induite par les pieds astrocytaires $[5,6]$. L'ouverture de cette barrière peut donc être provoquée par la rétraction des pieds astrocytaires. Cette ouverture crée une situation très anormale pour le tissu nerveux, qui, normalement, filtre les substan- qui se trouve en contact avec des protéines plasmatiques normalement exclues.

Dès les premières 24 heures, la mort neuronale s'accompagne donc de modifications importantes des cellules présentes dans la zone de lésion. En l'absence d'un recrutement significatif d'éléments cellulaires extérieurs, on assiste à une activation de la microglie résidente, à une altération morphologique des astrocytes entraînant la rupture de la glia limitans et à une ouverture de la barrière hémato-encéphalique. A ces modifications des éléments en place succède rapidement une seconde phase de recrutement et de prolifération cellulaire. En effet, pendant cette seconde phase, on observe le passage de macrophages à travers les cellules endothéliales activées (diapédèse). De plus, la rupture de la frontière du

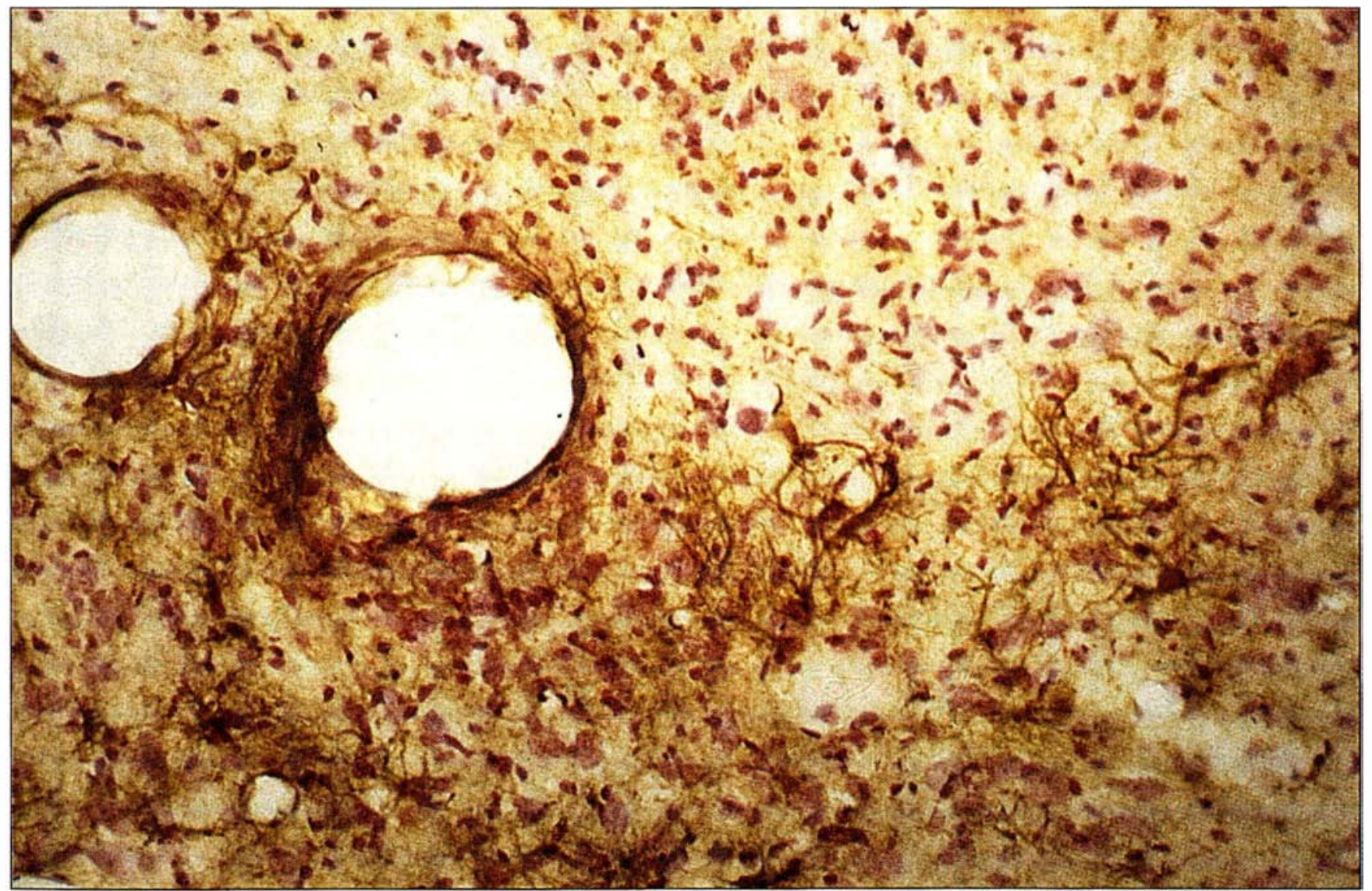

Figure 3. Réaction astrocytaire 4 jours après la lésion. Frontière entre la zone déplétée en neurones (en haut), caractérisée par une absence d'immunoréactivité à la GFAP, et le parenchyme thalamique non déplété en neurones (en bas), qui possède en revanche de nombreux astrocytes GFAP+ (le marquage brun révèle la fixation de l'anticorps dirigé contre la GFAP). 


\section{RÉFÉRENCES}

1. Peschanski M, Besson JM. Structural alteration and possible growth of afferents after kainate lesion in the adult thalamus. $J$ Comp Neurol 1987 ; 258 : 185-203.

2. Peschanski M, Isacson O. Fetal homotypic transplant in the excitotoxically neuron-depleted thalamus : light microscopy. J Comp Neurol 1988 ; 274 : 449-63.

3. Nothias F, Peschanski M. Homotypic fetal transplants into an experimental model of spinal cord neurodegeneration. J Comp Neurol $1990 ; 301$ : 520-34.

4. Streit WJ, Kreutzberg GW. Response of endogenous glial cells to motor neuron degeneration induced by toxic ricin. J Comp Neurol 1988 ; 268 : 248-63.

5. Janzer RC, Raff MC. Astrocytes induce blood-brain barrier properties in endothelial cells. Nature $1987 ; 325: 253-7$.

6. DeBault LE, Cancilla PA. GammaGlutamyl transpeptidase in isolated brain endothelial cells : induction by glial cells in vitro. Science $1980 ; 207$ : 653-5.

7. Matthews MA, Kruger L. Electron microscopy of non-neuronal cellular changes accompanying neural degeneration in thalamic nuclei of the rabbit. I. Reactive hematogenous and perivascular elements within the basal lamina. I Comp Neurol 1973 ; 148 : 285-312.

8. Imamoto $\mathrm{K}$, Leblond $\mathrm{CP}$ Presence of labeled monocytes, macrophages and microglia in a stab wound of the brain following an in jection of bone marrow cells labeled with $3 \mathrm{H}$-uridine into rats. J Comp Neurol $1977 ; 174: 255-79$

9. Murabe Y, Ibata Y, Sano Y. Morphological studies on neuroglia. III. Macrophage response and "Microgliocytosis" in kainic acid-induced lesions. Cell Tissue Res $1981 ; 218$ : 75-86.

10. Coffey PJ, Perry VH, Rawlins JNP. An investigation into the early stages of the inflammatory response following ibotenic acid-induced neuronal degeneration. Neurosci $1990 ; 35: 121-32$

11. Dusart I, Nothias F, Roudier F, Besson JM, Peschanski M. Vascularization of fetal cell suspension grafts in the excitotoxically lesioned adult rat thalamus. Dev Brain Res 1989 ; 48 : 215-28.

12. Shigematsu K, Kamo $\mathrm{H}$, Akiguchi I, Kimura J, Kamegama M, Kimura H. Neovascularization in kainic acid-induced lesions of rat striatum. An immunohistochemical study with laminin. Brain Res $1989 ; 501$ : système nerveux central permet l'entrée dans le parenchyme cérébral de cellules qui en sont normalement exclues.

\section{Phase 2 : recrutement et prolifération cellulaire}

Entre 4 et 15 jours après la mort neuronale, la densité cellulaire augmente considérablement dans la zone lésionnelle. Des amas de macrophages se forment le long de vaisseaux sanguins (figure 2a), comme cela a été décrit dans d'autres cas de lésion [7-10], et des images suggérant l'existence d'une diapédèse ont été observées en microscopie électronique. De plus, des cellules fixant la lectine (macrophages recrutés ou cellules microgliales résidentes) sont observées en phase de mitose dans la zone lésionnelle (figure $2 b$ ). L'augmentation de la densité cellulaire est donc due, au moins en partie, à un recrutement de monocytes et à la prolifération in situ de ces cellules recrutées ou des cellules microgliales résidentes.

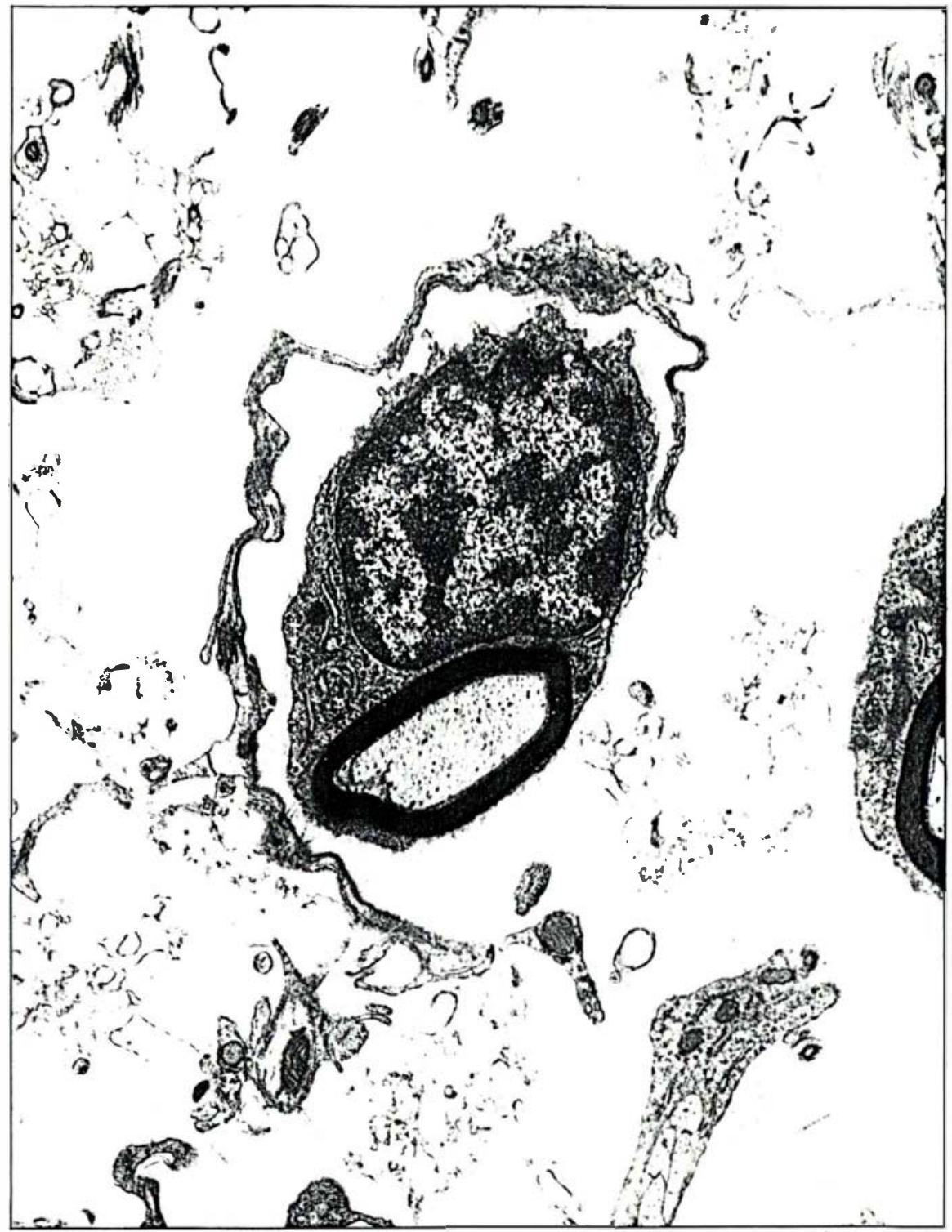

Figure 4. Remyélinisation par des cellules de Schwann. Cette photo prise à l'aide d'un microscope électronique (G $\times 7000$ ) montre un axone myélinisé par une cellule de Schwann. Lorsqu'un axone est myélinisé par une cellule de Schwann il existe une couche de cytoplasme autour de cet axone dans laquelle se trouve le noyau de la cellule de Schwann, une lame basale entoure l'ensemble formé par la cellule de Schwann et l'axone. 


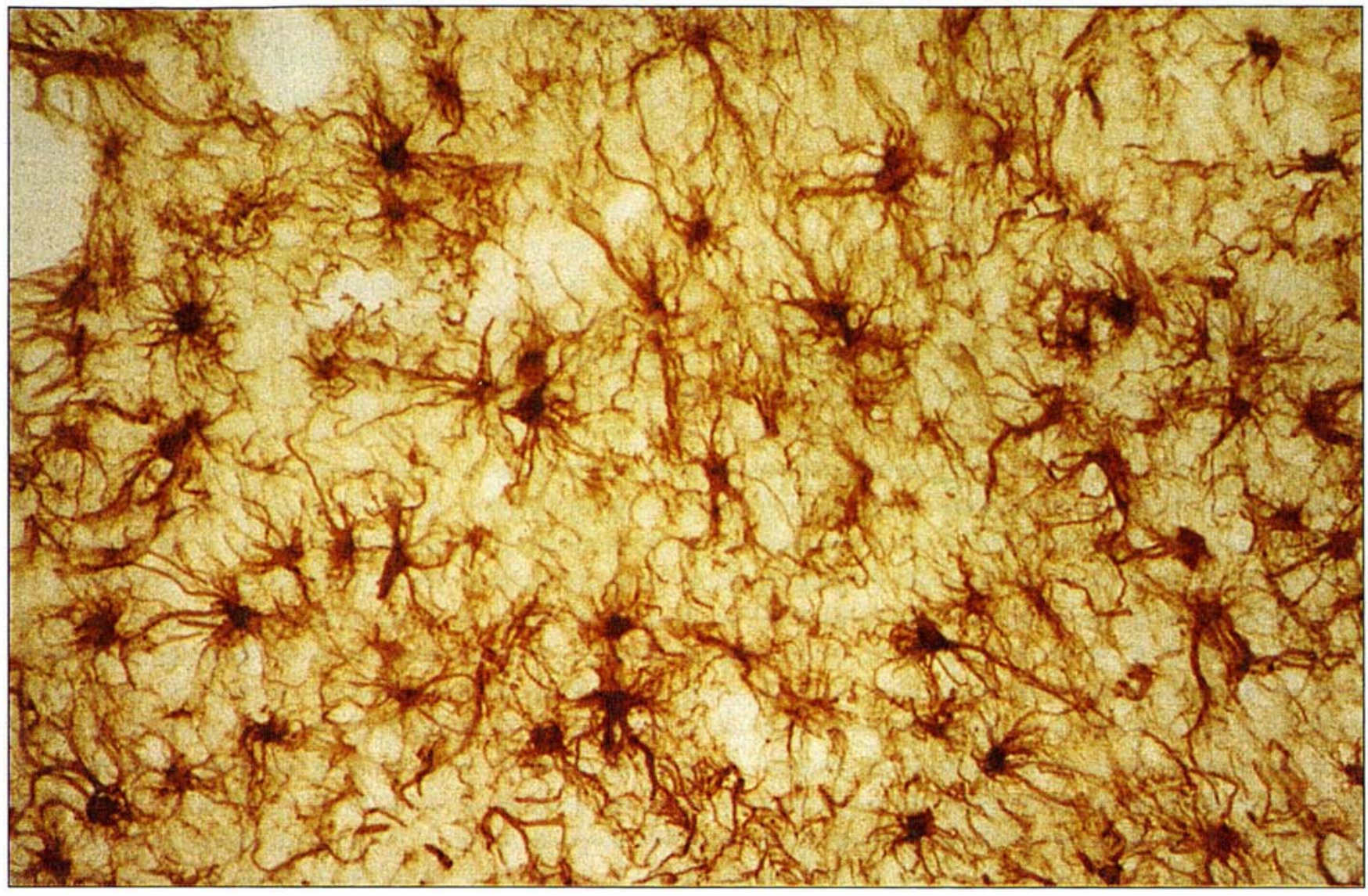

Figure 5. Astrocytes dans la zone de déplétion neuronale 1 mois après la lésion. Les astrocytes ont une forme étoilée, ils sont hypertrophiés et fortement immunoréactifs pour la GFAP lle marquage brun révèle la fixation de l'anticorps dirigé contre la GFAP).

On observe, également, une angiogenèse importante, avec un développement de la microvascularisation [11] mais aussi des artérioles (vaisseaux dont la paroi contient une couche de fibres musculaires lisses). Ces résultats sont cohérents avec l'augmentation décrite de la concentration en laminine, un composant de la matrice extracellulaire des vaisseaux [12]. On peut suggérer que les macrophages activés jouent un rôle important dans cette angiogenèse réactive [13], en particulier par leur sécrétion de TNF (tumor necrosis factor) et d'IL-1 qui possèdent un fort pouvoir angiogénétique $[14,15]$.

Dans le même temps, au contraire, le marquage immunocytochimique de la GFAP ne permet plus de visualiser des cellules astrocytaires dans la zone lésionnelle (figure 3), un phénomène déjà remarqué par d'autres $m / s n^{\circ} 8$, vol. 7 , octobre 91 auteurs [16-18]. En microscopie électronique, ces cellules ont un cytoplasme pauvre en gliofilaments, ce qui peut expliquer leur absence d'immunoréactivité. Certaines présentent des signes de dégénérescence. Les astrocytes ne semblent donc pas participer à la gliose durant cette période.

Jusqu'à 15-20 jours après la lésion, le tissu est également caractérisé par une intense démyélinisation. Les axones, quel que soit leur diamètre, sont dépourvus de myéline. De telles images ont été décrites dans les lésions purement démyélinisantes, notamment dans la sclérose en plaques*. $\mathrm{Au}$ vu de données récentes de la littérature, deux mécanismes pourraient expliquer cette démyélinisation. D'une part, l'ouverture de la barrière

* Voir l'article de M. Gumpel, p. 782 hémato-encéphalique permet aux facteurs circulants du système du complément de pénétrer dans le parenchyme cérébral. On a décrit une activation de ce système par les lipopolysaccharides contenus dans les oligodendrocytes et la myéline [19, 20]. D'autre part, les macrophages activés produisent du $\mathrm{TNF} \alpha$; cette cytokine est toxique pour les oligodendrocytes et la myéline du système nerveux central [21].

A partir de la troisième semaine, on observe une remyélinisation des axones par des oligodendrocytes, mais aussi par des cellules de Schwann [22] (figure 4). Cette observation est surprenante dans la mesure où les cellules de Schwann sont caractéristiques des nerfs périphériques mais n'existent pas, normalement, dans le système nerveux central. Parmi les axones périphériques qu'elles entou- 


\section{RÉFÉRENCES}

13. Beck DW, Hart MN, Cancilla PA. The role of the macrophage in microvascular regeneration following brain injury. $J$ Neuropathol Exp Neurol 1983 ; 42 : 601-14

14. Polverini PJ, Leibovich SJ. Induction of neovascularization in vivo and endothelial proliferation in vitro by tumor-associated macrophages. Lab Invest 1984 ; 51 : 635-42.

15. Giulian D, Woodward J, Young DG, Krebs JF, Lachman LB. Interleukin-1 injected into mammalian brain stimulates astrogliosis and neovascularization. $J$ Neurosci 1988 ; 8 : 2485-90.

16. Björklund $\mathrm{H}$, Olson L, Dahl D, Schwarcz R. Short- and long-term consequences of intracranial injections of the excitotoxin, quinolinic acid, as evidenced by GFAP immunohistochemistry of astrocytes. Brain Res 1986 ; 371 : 267-77.

17. Eriksdotter-Nilsson $\mathrm{M}, \mathrm{B}$ jorklund $\mathrm{H}$, Dahl D, Olson L, Ingvar M. Sustained seizures cause circumscribed cerebral changes in glial fibrillary acidic protein, neurofilament and laminin fluorescence. Exp Brain Res 1987 ; 69 : 155-66

18. Ogawa M, Araki M, Nagatsu I, Yoshida M. Astroglial cell alteration caused by neurotoxins : immunohistochemical observations with antibodies to glial fibrillary acidic protein, laminin, and tyrosine hydroxylase. Exp Neurol 1989 ; 106 : 187-96

19. Scolding NJ, Morgan BP, Houston WAJ, Linington C, Campbell AK, Compston DAS. Vesicular removal by oligodendrocytes of membrane attack complexes formed by activated complement Nature 1989 ; 339 : 620-2.

20. Wren DR, Noble M. Oligodendrocytes and oligodendrocyte/type-2 astrocyte progenitor cells of the adult rats are specifically susceptible to the lytic effects of complement in absence of antibody. Proc Natl Acad Sci USA 1989 ; 86 : 9025-9.

21. Selmaj KW, Raine CS. Tumor necrosis factor mediates myelin and oligodendrocyte damage in vitro. Ann Neurol 1988 ; 23 339-46.

22. Dusart I, Isacson O, Nothias F, Gumpel M, Peschanski M. Presence of Schwann cells in neurodegenerative lesions of the central nervous system. Neurosci Lett 1989 ; $105: 246-50$

23. Blakemore WF. Remyelination of dernyelinated spinal cord axons by Schwann cells. Spinal Cord Reconstruction. Kao CC, Bunge RP Reier PJ, eds. New York: Raven Press,
24. Harrison BM. Schwann cells divide in a demyelinating lesion of the central nervous system. Brain Res 1987 ; 409 : 163-8.

25. Sims TJ, Gilmore SA. Interactions between intraspinal Schwann cells and the cellular constituents normally occurring in the spinal cord : an ultrastructural study in the irradiated rat. Brain Res $1983 ; 276$ : 17-30.

26. Blakemore WF, Patterson RC. Observations on the interactions of Schwann cells and astrocytes following $\mathrm{X}$-irradiation of neonatal rat spinal cord. $J$ Neurocytol 1975 ; $4: 573-85$.

27. Perry VH, Hayes L. Lesion-induced myelin formation in the retina. $J$ Neurocytol 1985 ; 14 : 297-307.

28. Baichwal RR, Bigbee JW, DeVries GH. Macrophage-mediated myelinrelated mitogenic factor for cultured Schwann cells. Proc Natl Acad Sci USA 1988 ; 85 : 1701-5.

29. Salzer JL, Williams AK, Glaser L Bunge RP. Studies of Schwann cell proliferation. II. Characterization of the stimulation and specificity of the response to a neurite membrane fraction. J Cell Biol 1980b ; 84 : 753-66.

30. Raine CS, Cross AH. Axonal dystrophy as a consequence of long-term demyelination. Lab Invest 1989 ; 60 : 714-15.

31. Assouline JG, Bosch P, Lim R, Kim IS, Jensen R, Pantazis NJ. Rats astrocytes and Schwann cells in culture synthesize nerve growth factor-like neuritepromoting factors. Dev Brain Res 1987 ; 31 : 103-18.

32. Brachet $P$. Le facteur de croissance nerveuse NGF : rôle dans la plasticité et la maintenance fonctionnelle de la cellule neuronale. médecine/sciences $1990 ; 6$ : 854 .

33. Reier PJ. Gliosis following CNS injury : the anatomy of astrocytic scars and their influences on axonal elongation. In : Fedoroff S, Vernadakis A, eds. Astrocytes, Cell Biology and Pathology of Astrocyte, vol. 3. New York: Academic Press, 1986: 263-324.

34. Maxwell DS, Kruger L. The fine structure of astrocytes in cerebral cortex and their response to focal injury produced by heavy ionizing particles. J Cell Biol 1965 ; $25: 141-57$

35. Bignami A, Dahl D. The astroglial response to stabbing. Immunofluorescence studies with antibodies to astrocyte-specific pro tein (GFAP) in mammalian and submammalian vertebrates. Neuropathol Appl Neurobiol 1976 ; 2 : 99-110. rent, on trouve toutefois ceux qui innervent les parois des artérioles du cerveau $[23,24]$. L'angiogenèse d'artérioles décrite au-dessus conduit donc vraisemblablement des cellules de Schwann à proximité des zones lésionnelles. Elles sont normalement exclues du système nerveux central par les pieds astrocytaires qui forment la glia limitans $[23,25]$. La rupture de la glia limitans permet le passage des cellules de Schwann dans la lésion [26, 27]. Enfin, les macrophages ayant phagocyté de la myéline [28] et la présence d'axones démyélinisés [29] favorisent la prolifération des cellules de Schwann et pourraient donc promouvoir leur présence dans le parenchyme.

En conclusion, la deuxième phase de réponse gliale et vasculaire à la mort neuronale est marquée par l'abondance des cellules macrophagiques. Nous avons vu que ces cellules pourraient jouer non seulement le rôle d'éboueurs, mais aussi celui de "pôle organisateur " de réponses cellulaires variées.

C'est également durant cette seconde phase qu'apparaissent les modifications morphologiques des terminaisons axonales privées de leurs cibles. On peut suggérer que l'élargissement terminal des axones est lié à la démyélinisation [30]. Dans un second temps, les cellules de Schwann pourraient promouvoir la persistance des afférences axonales. Dans certaines conditions, ces cellules acquièrent en effet un phénotype activé, caractérisé par une capacité à sécréter des substances trophiques [31] parmi lesquelles figure le nerve growth factor (facteur de croissance nerveux [32]).

\section{Phase 3 : établissement de la cicatrice astrocytaire}

La troisième phase, qui commence un mois après la mort neuronale, se distingue de la phase précédente par deux caractéristiques : les astrocytes occupent l'ensemble de la lésion (figure 5) tandis que le nombre des autres éléments cellulaires diminue. Cette phase constitue l'établissement de la cicatrice astrocytaire.

Ce n'est qu'à partir de 10-15 jours après la mort neuronale que des astrocytes immunoréactifs pour la GFAP réapparaissent dans la lésion. 


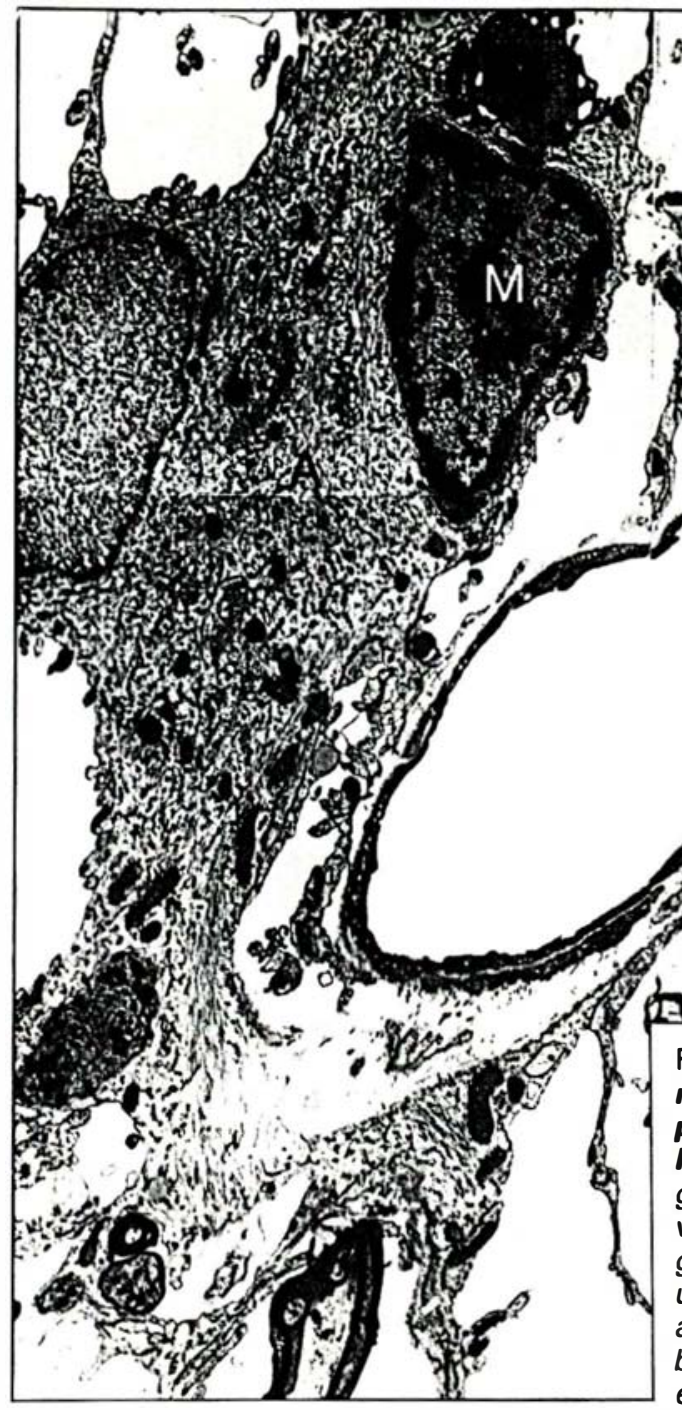

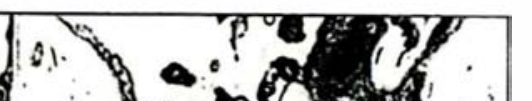
$\operatorname{los}$ (n) 10

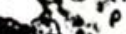

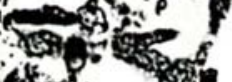
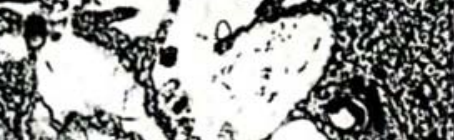
- 1.00
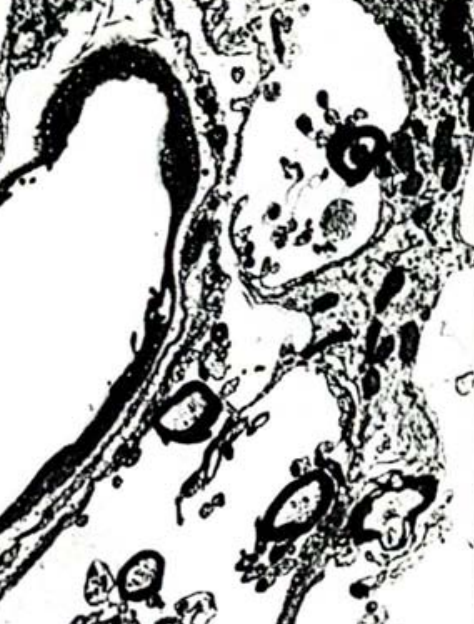

a.

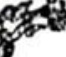

Figure 6. Photographie en microscopie électronique du parenchyme lésé 1 an après la lésion (G $\times$ 7 000). Un prolongement astrocytaire entoure un vaisseau et une cellule microgliale/macrophagique (M). (A) est un astrocyte. Le prolongement astrocytaire forme une lame basale du côté de la cellule endothéliale, il reforme ainsi la glia limitans.

Les astrocytes sont alors peu nombreux, leur nombre augmentant rapidement au-delà d'un mois. Ces astrocytes présentent un phénotype dit "réactif": leur corps cellulaire est large, leurs prolongements épais et ils sont très immunoréactifs pour la GFAP. Dès leur entrée dans la zone de lésion, les astrocytes sont souvent associés aux vaisseaux sanguins qu'ils entourent de prolongements, reformant une lame basale du côté bordant les cellules endothéliales. Nos observations correspondent à de nombreuses autres qui suggèrent que les astrocytes tendent à reconstruire toute brèche apparue dans la glia limitans [33]. Cette reconstruction $\mathrm{m} / \mathrm{s} n^{\circ} 8$, vol 7 , octobre 91
Parallèlement, le nombre des éléments non astrocytaires diminue dans la lésion. Cela semble correspondre à la cicatrice gliale dont on sait qu'elle est réalisée par les astrocytes [33-35].

En conclusion, la mort neuronale produite par l'injection de substances excitotoxiques provoque, dans un premier temps, des modifications des cellules en place avant la lésion, conduisant notamment à la disparition de la frontière du système nerveux central. Au cours des semaines suivantes, différentes populations cellulaires pénètrent dans le parenchyme lésé (macrophages, cellules de Schwann). La reconstruction d'une frontière entre le tissu cérébral et le reste de l'organisme par les astrocytes aboutit ensuite, à long terme, à la constitution d'une cicatrice stérile. Ces modifications influent probablement sur les afférences axonales privées de leurs cibles par la lésion excitotoxique. Les tentatives régénératives semblent être favorisées lors de l'étape de prolifération et de recrutement cellulaire (modifications morphologiques persistantes des afférences axonales, présence de cellules de Schwann), puis tendent à disparaître. Il faudrait, pour mieux comprendre le rôle des différents types cellulaires, essayer de perturber un des éléments de réponse à la lésion et voir dans quel sens les autres éléments évoluent de la frontière du système nerveux central se fait autour des vaisseaux sanguins (figure 6), mais aussi autour des éléments étrangers présents dans la lésion. Les cellules de Schwann sont, ainsi, individuellement entourées de prolongements astrocytaires formant une lame basale.

A long terme, tous les éléments cellulaires sont entourés par des astrocytes. Cependant les astrocytes respectent toujours la différence entre les éléments du système nerveux central et les autres. En effet, ils ne forment pas de lame basale lorsqu'ils entourent des oligodendrocytes et les axones qu'ils myélinisent, ou les cellules microgliales/macrophagiques.

\section{Summary}

Glial and vascular responses to excitotoxically-induced neuronal death

This study was undertaken to follow the time course of changes in the morphology and distribution of glial cells that take place during the formation of a glial scar after injection of kainic acid in the thalamus of the rat. The progression of the glial reaction to the lesion was followed from 24 hours to 18 months after the kainate injection. The microglial/macrophagic cells were labeled with the B4 iso- 
Summary (suite de la p. 797)

lectin from Griffonia simplicifolia. The astrocytes were identified using an antibody raised against glial fibrillary acidic protein (GFAP). The vascularization was followed both in terms of morphology of the capillaries and of the integrity of the blood-brain-barrier (BBB). This analysis was performed using perfusion of blood vessels with Indian Ink for the study of the morphology, and circulation of HRP for the BBB. We have studied the fine structure of Schwann cells and oligodendrocytes.

Three periods could be distinguished during the evolution of the cellular response in the neurondepleted area. The first one is the initial response of elements present before the kainate injection. It consists of a morphological modification in the astrocytes, a rupture of the glia limitans, an opening of the $\mathrm{BBB}$, and an activation of resident microglial cells. The second period is characterized by a cellular recruitment and proliferation. Blood monocytes enter the neuron-depleted area. There is an angiogenesis, a demyelination of axons, an appearance of growth cone like structures and the recruitment of Schwann cells. The last period leads to the formation of the glial scar by astrocytes.

We have tried to show that the different events do not evolve independently but that the injection of kainate and the neuronal death lead to a series of cellular interactions, which in turn results in a glial scar. It may be that attempts at regeneration find a favorable environment during the period of cellular proliferation which is terminated by the formation of the glial scar (after one month) and the diminution of the number of the other elements.

TIRÉS A PART 